\title{
Intensity of microwave signals expected from molecular Bremsstrahlung radiation in extensive air showers
}

\author{
Imen AI Samarai* \\ LPNHE, CNRS/IN2P3 \& Université Pierre et Marie Curie, Paris, France \\ E-mail: ialsamarelpnhe.in2p3.fr \\ Olivier Deligny \\ IPNO, CNRS/IN2P3 \& Université Paris X, Paris, France \\ E-mail: delignyeipno.in2p3.fr
}

\begin{abstract}
A potential detection technique of ultra-high energy cosmic rays would be the use of the molecular Bremsstrahlung radiation emitted by low-energy electrons left after the passage of the showers in the atmosphere. The emission mechanism is expected from quasi-elastic collisions of electrons produced in the shower by the ionisation of the molecules in the atmosphere. Given the low energy of the ionisation electrons, $\mathrm{GHz}$ photons resulting from this emission mechanism are expected to be emitted isotropically. This would allow for mapping the ionisation content along the showers through the intensity of the microwave signals detected at ground, in the same way as done for the fluorescence technique. In this contribution, a detailed calculation of the spectral intensity of photons at ground level originating from the transitions between unquantised energy states of free ionisation electrons is presented. The obtained spectral intensity is shown to be $\simeq$ $4.010^{-26} \mathrm{~W} \mathrm{~m}^{-2} \mathrm{~Hz}^{-1}$ at $10 \mathrm{~km}$ from the shower core for a vertical shower induced by a proton of $10^{17.5} \mathrm{eV}$. The detectability of the derived spectral intensities with the current experimental setups is discussed.
\end{abstract}

The 34th International Cosmic Ray Conference,

30 July- 6 August, 2015

The Hague, The Netherlands

${ }^{*}$ Speaker. 


\section{Introduction}

The measurement of the composition of high energy cosmic rays (CRs) as a function of energy is one of the main challenges that $\mathrm{CR}$ observatories are currently facing. Composition measurements are essentially given by the fluorescence technique, by means of fluorescence light detectors that observe the longitudinal profile of the extensive air showers (EAS) through a calorimetric measurement during their development in the atmosphere. The average and the RMS of the atmospheric depth $X_{\max }$ at which the shower reaches its maximum development are excellent indicators of the CR primary particle. However, due to the nature of the fluorescence emission, this technique has substantial limitations as it requires clear and moonless nights, reducing the measurement duty cycle to $\simeq 10-15 \%$. In addition, the scarcity of particles at the highest energies results in the absence of composition measurements of CRs with energies greater than $\simeq 310^{19} \mathrm{eV}$.

The measurement in laboratory [1] of low energy electrons emitting in the $\mathrm{GHz}$ frequency band attributed to molecular Bremsstrahlung radiation (MBR), has triggered ground-based experiments aiming at the detection of this radiation from extensive air showers. Comparing to the fluorescence radiation, the nature of the MBR radiation potentially offers a full duty-cycle. Furthermore, it is not absorbed in the atmosphere and its expected isotropic and unpolarized nature, would allow the mapping of the ionisation content, scaling linearly with energy, through the intensity of the microwave signals detected at ground.

Based on the studies reported in [2], we first present in section 2 a macroscopic model of MBR radiation in the frame of free-free transitions, emitted from low energy electrons created after the passage of the EAS relativistic electrons. In section 3, the expected signal at ground from a reference shower is presented. Finally, results are discussed in section 4.

\section{Microwave emission from MBR in extensive air showers}

An extensive air shower is considered as a thin plane front of high energy charged particles propagating in the atmosphere at the speed of light $c$. After the succession of a few initial steps in the cascade, all showers can be described by reproducible macroscopic states. In particular, the total number of primary $\left(e^{+} / e^{-}\right) \mathrm{N}_{e, p}$ at each altitude $a$, can be adequately parameterised by the Gaisser-Hillas function [3]. The lateral extension of the core depends on the mean free path and can be expressed in terms of the Molière radius $\mathrm{R}_{M}$ such that $90 \%$ of the energy is contained within a distance $r$ from the axis smaller than $R_{M}$. The NKG lateral distribution function denoted hereafter by $g(r, a)$ reproduces well the observations [4]. The number of primary $\left(e^{+} / e^{-}\right)$per unit surface, $n_{e, p}(r, a)$, is then simply obtained by folding the longitudinal profile to the normalized lateral one. For a vertical shower, $n_{e, p}(r, a)$ reads as:

$$
n_{e, p}(r, a)=N_{e, p}(a) \frac{g(r, a)}{2 \pi \int \mathrm{d} r \operatorname{rg}(r, a)} .
$$

Through their passage in the atmosphere, the charged particles of an EAS deposit their energy mainly through ionisation. Under the hypothesis that the resulting ionisation electrons are emit- 
ted isotropically ${ }^{1}$, the instantaneous flux of ionisation electrons per kinetic energy band can be described by the expression:

$$
\phi_{e, i}^{0}\left(r, a, T_{e}\right)=\frac{c \beta\left(T_{e}\right) f_{0}\left(T_{e}\right)}{2\left\langle I_{0}+T_{e}\right\rangle}\left\langle\frac{\mathrm{d} E}{\mathrm{~d} X}\right\rangle \rho_{m}(a) n_{e, p}(r, a) .
$$

with $I_{0}$ the ionisation potential to create an electron-ion pair in air $\left(I_{0}=15.6 \mathrm{eV}\right.$ for $\mathrm{N}_{2}$ and $I_{0}=$ $12.1 \mathrm{eV}$ for $\mathrm{O}_{2}$ molecules). The bracketed expression $\left\langle\frac{\mathrm{d} E}{\mathrm{~d} X}\right\rangle$ stands for the mean energy loss of primary electrons per grammage unit, almost independent of the primary electron energy over a range of few tens of $\mathrm{MeV}$. The normalised function $f_{0}\left(T_{e}\right)$ is the distribution in kinetic energy of the resulting ionisation electrons, experimentally determined and accurately parameterised for primary electrons with kinetic energies $T_{e}^{p}$ up to several keV [5]. At higher energies, relativistic effects as well as indistinguishability between primary and secondary electrons have been shown to modify the low-energy electrons behaviour. We thus adopt an analytical formulation given in [6] where $T_{e}$ ranges from 0 to $T_{e}^{\max }=\left(T_{e}^{p}-I_{0}\right) / 2$, expressed as:

$$
f_{0}\left(T_{e}\right)=K \frac{1+C \exp \left(-T_{e} / T_{k}\right)}{T_{e}^{2}+\bar{T}^{2}} .
$$

The constant $C$ is determined in the same way as in [5] so that $\int \mathrm{d} T_{e} C /\left(T_{e}^{2}+\bar{T}^{2}\right)$ reproduces the total ionisation cross section, $T_{k}=77 \mathrm{eV}$ is a parameter acting as the boundary between close and distant collisions, the constant $K$ is tuned to guarantee $\int \mathrm{d} T_{e} f_{0}\left(T_{e}\right)=1$, and $\bar{T}=13.0(17.4) \mathrm{eV}$ for nitrogen (oxygen). In the energy range of interest, this expression leads to $\left\langle T_{e}\right\rangle \simeq 40 \mathrm{eV}$, in agreement with the well-known stopping power.

By determining the time evolution of the energy of the ionisation electrons, the fraction of time during which these electrons can emit MBR can be assessed. The evolution in time of $\phi_{e, i}^{0}\left(r, a, T_{e}\right)$ can be fully encompassed in the time dependence of the distribution in kinetic energy $f\left(T_{e}, t\right)$ of the ionisation electrons. A Boltzmann equation describing the processes that these secondary electrons undergo considers collision terms in the expression of ionisation, excitation (including ro-vibrational excitation) of electronic levels, and attachment:

$$
\begin{aligned}
\frac{\partial f}{\partial t}\left(T_{e}, t\right) & =-n_{m}(a) c \beta\left(T_{e}\right)\left(\sigma_{\mathrm{att}}\left(T_{e}\right)+\sigma_{\mathrm{exc}}\left(T_{e}\right)+\sigma_{\mathrm{ion}}\left(T_{e}\right)\right) f\left(T_{e}, t\right) \\
& +n_{m}(a) c \int_{T_{e}}^{T_{e}^{\max }} \mathrm{d} T_{e}^{\prime} \beta\left(T_{e}^{\prime}\right)\left(\frac{\mathrm{d} \sigma_{\mathrm{ion}}}{\mathrm{d} T_{e}}\left(T_{e}^{\prime}, T_{e}\right)+\frac{\mathrm{d} \sigma_{\mathrm{ion}}}{\mathrm{d} T_{e}}\left(T_{e}^{\prime}, T_{e}^{\prime}-T_{e}\right)\right) f\left(T_{e}^{\prime}, t\right) \\
& +n_{m}(a) c \int_{T_{e}}^{T_{e}^{\max }} \mathrm{d} T_{e}^{\prime} \beta\left(T_{e}^{\prime}\right) \frac{\mathrm{d} \sigma_{\mathrm{exc}}}{\mathrm{d} T_{e}}\left(T_{e}^{\prime}, T_{e}\right) f\left(T_{e}^{\prime}, t\right),
\end{aligned}
$$

where $\sigma_{i}$ denotes the cross sections of interest (ionisation, excitation and attachment processes), and $n_{m}(a)$ is the density of molecules at an altitude $a$. The first term in the right hand side stands for the disappearance of electrons with kinetic energy $T_{e}$, while the second and third terms stand for the appearance of electrons with kinetic energy $T_{e}$ due to ionisation and excitation reactions

\footnotetext{
${ }^{1}$ We neglect the fact that they should be produced along the flow of the general motion of the high-energy electrons of the cascade. As low-energy photons will be emitted isotropically from these non-relativistic electrons, this assumption does not impact any of the results concerning the incoherent emission of photons.
} 
initiated by electrons with higher kinetic energy $T_{e}^{\prime}$. The electron attachment process with oxygen is precisely depicted in [7], while the ionisation cross-section as well as the excitation of atomic levels of the $\mathrm{O}_{2}$ and $\mathrm{N}_{2}$ molecules are taken from reference [8] and [9] respectively.
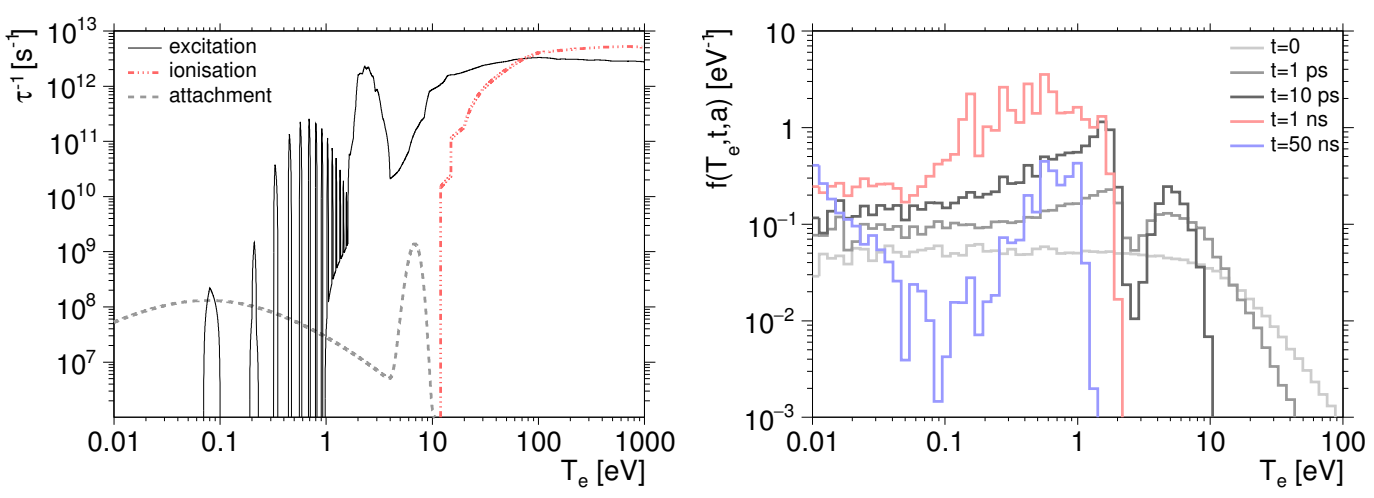

Figure 1: Left: Collision rates of interest as a function of electron kinetic energy, at sea level. Right: Distribution in kinetic energy of the ionisation electrons at different times at sea level.

The collision rate $\tau^{-1}$ as a function of energy through each collision process at sea level is shown in the left panel of figure 1. Few important remarks can be noted for the following:

- $E>100 \mathrm{eV}$ : Ionisation is the dominant energy loss process.

- $1.7 \mathrm{eV}^{2}<E<100 \mathrm{eV}$ : Excitation is the dominant energy loss process.

- $E<1.7 \mathrm{eV}$ : Ro-vibrational excitation reactions compete within some quantised energy ranges with the attachment process which causes a real disappearance of electrons.

The characteristic timescales of each process taking place are determined from the collision rates. Within the $\mathrm{eV}$ energy range, the attachment process is performant at timescales of $\simeq 20 \mathrm{ns,}$ while the timescale between two successive ionisations is below $\simeq 1 \mathrm{ps}$, and the excitation of atomic levels is also of the order of $\simeq 1$ ps. Solving the Boltzmann equation given in equation 2.4 reveals the evolution with time of the function $f$, obtained by Monte-Carlo for electrons produced at sea level. At different time stamps, we calculate the energy distribution of the population of electrons. This is illustrated in the right panel of figure 1 . It is noteworthy that after $\simeq 1 \mathrm{~ns}$, all electron energies get below the lowest excitation threshold belonging to the continuous spectrum of energy losses at $1.7 \mathrm{eV}$, resulting in a sharp cut-off in the $f$ function. For even longer durations, free electrons can still collide through excitation processes when their energies resonate with the corresponding quantised reactions, while the overall population starts to disappear through the attachment process.

An emission of continuum Bremsstrahlung radiation can be produced by the ionisation electrons through quasi-elastic scattering with molecular nitrogen and, to smaller extent, oxygen, as long as they remain free.

$$
e^{ \pm}+M \rightarrow e^{ \pm}+M+\gamma
$$

In this approach, the production of photons with energies $h v$ corresponds to transitions between unquantised energy states of the free electrons ('free-free' transitions). The spectral intensity

\footnotetext{
${ }^{2}$ Threshold to excite one of the electronic levels of oxygen
} 
at ground level can thus be deduced from the collision rate of ionisation electrons with neutral molecules in air. Because the production rate $r_{\gamma}$ of photons with energy $h v$ per volume unit can be considered proportional to the target density, it can thus be retrieved from the electron flux and the free-free cross section $\sigma_{f f}\left(T_{e}, h v\right)$, leading to the following expression:

$$
r_{\gamma}(r, a, t, v)=n_{m}(a) \int_{0}^{T_{e}^{\max }} \mathrm{d} T_{e} \phi_{e, i}\left(r, a, T_{e}, t\right) \sigma_{\mathrm{ff}}\left(T_{e}, h v\right)
$$

The free-free cross-section as obtained in reference [10] can be related to the electron momentum transfer cross-section through:

$$
\sigma_{\mathrm{ff}}\left(T_{e}, h v\right)=\frac{4}{3 \pi} \frac{\alpha^{3}}{R_{y}}\left(1-\frac{h v}{2 T_{e}}\right) \sqrt{1-\frac{h v}{T_{e}}} T_{e} \sigma_{m}\left(T_{e}\right)
$$

with $\alpha$ the fine-structure constant and $R_{y}$ the Rydberg constant. Since electrons have kinetic energies in the range of few $\mathrm{eV}$ and photons in the $\mathrm{GHz}$ energy range, this expression is almost independent of $h v$ and can be reduced to $\sigma_{\mathrm{ff}}\left(T_{e}\right)=1.21110^{-8} T_{e} \sigma_{m}\left(T_{e}\right)$. The electron momentum transfer cross-section has been well measured on various targets. Compiled tables provided in reference [9] were used for the following.

Hence, from the knowledge of the collision rate per volume unit $r_{\gamma}$, the emitted power per volume unit at each point $(r, a)$ can be simply obtained by coupling this rate to the energy of the emitted photons, so that the emitted spectral power per volume unit can be written as:

$$
\begin{aligned}
\frac{\mathrm{d}^{2} P}{\mathrm{~d} v \mathrm{~d} V}(r, a, t) & =\frac{\mathrm{d}}{\mathrm{d} \nu}\left(h v r_{\gamma}(r, a, t)\right) \\
& =\frac{h c \rho_{m}^{2}(a) \mathscr{N}_{A}}{2 A\left(I_{0}+\left\langle T_{e}\right\rangle\right)}\left\langle\frac{\mathrm{d} E}{\mathrm{~d} X}\right\rangle n_{e, p}(r, a) \tilde{\sigma}(t, a),
\end{aligned}
$$

where $\tilde{\sigma}(t, a)$ is an effective cross-section defined as:

$$
\tilde{\sigma}(t, a)=\int_{0}^{T_{e}^{\max }} \mathrm{d} T_{e} f\left(T_{e}, t, a\right) \beta\left(T_{e}\right) \sigma_{\mathrm{ff}}\left(T_{e}\right) .
$$

The radiation produced by individual electrons-nitrogen/oxygen encounters can be considered here to pass the interaction volume without absorption or reflection. The validity of this assumption has been justified in [2]. At any distance $R$, the spectral intensity received from sources contained in an infinitesimal volume $\mathrm{d} V$ is proportional to $\mathrm{d}^{2} P / \mathrm{d} v \mathrm{~d} V$ times $\mathrm{d} V$ and weighted by $4 \pi R^{2}$ given that photons are emitted isotropically ${ }^{3}$ from each source. In this way, the observable spectral intensity at any ground position $\mathbf{x}_{g}, \Phi_{g}$, is simply the sum of the uncorrelated contributions of individual encounters:

$$
\Phi_{g}\left(\mathbf{x}_{g}, t\right)=\int_{0}^{\infty} r \mathrm{~d} r \int_{0}^{2 \pi} \mathrm{d} \varphi \int_{0}^{\infty} \mathrm{d} a \frac{1}{4 \pi R^{2}(r, \varphi, a)} \frac{\mathrm{d}^{2} P}{\mathrm{~d} \nu \mathrm{d} V}\left(r, a, t_{d}(t, r, \varphi, a)\right) .
$$

Here, $R$ is the distance between the position at ground $\mathbf{x}_{g}$ and the position of the current source in the integration, and $t_{d}$ is the delayed time at which the emission occurred. Fixing the reference

\footnotetext{
${ }^{3}$ The assumption on isotropy is justified for non-relativistic electrons in the regime where the energy of the photons is low compared to the energy of the electrons [11], as this is the case here.
} 
time $t_{0}$ to the time at which the shower front crosses the ground, each source at altitude $a$ started emitting radiation at the time the shower front passed at that altitude (i.e. at $\left.t_{0}-a / c\right)$. Each photon crossing the ground at time $t$ (with the condition that $t \geq t_{0}$ ) coming from a source at altitude $a$ and located at a distance $r$ from the shower axis was emitted at time $t-R n(a, v) / c$ - with $n(a, v)$ the refractive index of the atmosphere integrated along the line of sight between the emission point and the observer. The delayed time $t_{d}(t, r, \varphi, a, v)$, denoted hereafter $t_{d}$ only for convenience, is thus expressed as:

$$
t_{d} \equiv t_{d}(t, r, \varphi, a, v)=t-t_{0}-\left(\frac{R(r, \varphi, a) n(a, v)}{c}-\frac{a}{c}\right) .
$$

Requiring that emissions occur only at $t_{d} \geq 0$, a Heaviside function denoted $\Theta$ is introduced leading to the following semi-analytical expression for the observable spectral intensity:

$$
\Phi_{g}\left(\mathbf{x}_{g}, t\right)=\frac{h c \mathscr{N}_{A}}{8 \pi A\left(I_{0}+\left\langle T_{e}\right\rangle\right)}\left\langle\frac{\mathrm{d} E}{\mathrm{~d} X}\right\rangle \int_{0}^{\infty} r \mathrm{~d} r \int_{0}^{2 \pi} \mathrm{d} \varphi \int_{0}^{\infty} \mathrm{d} a \frac{\rho_{m}^{2}(a) n_{e, p}(r, a)}{R^{2}(r, \varphi, a)} \tilde{\sigma}\left(t_{d}, a\right) \Theta\left(t_{d}\right) .
$$

An independent estimation of the radiated power using the classical field theory formalism can be applied, resulting in the same predictions found using the 'free-free approach'. Detailed expressions in this frame can be found in the Appendix of reference [2].

Possible attenuation and absorption effects that ionisation electrons can experience via inverse Bremsstrahlung and stimulated Bremsstrahlung within the electrons-neutral molecules plasma are discussed in reference [2]. The estimation is based on the calculation of the absorption coefficient $\alpha_{v}$ defined as the relative attenuation per unit length of the emitted photons. It has been found that close to the shower core and to the maximum of shower development (that is, within the denser plasma region), this leads to $v^{2} \alpha_{v} \simeq 10^{-4} \mathrm{~m}^{-1} \mathrm{~Hz}^{2}$. At GHz frequencies, the absorption can thus be considered as negligible. Moreover, suppression effects that may affect the spectral intensity given in equation 2.13 can arise from successive collisions, known as the plasma dispersion effect [12], leading to destructive interferences of the radiated fields. To assess the impact of this effect, we linearise a simplified Boltzmann equation pertaining only to the case of a distribution function $f\left(T_{e}\right)$ stationary in time in the absence of any emitted radiation. In this case, it can be shown that the plasma dispersion effects result in a suppression factor in the integrand of $\tilde{\sigma}$ defined in equation 2.10 reading as [12]:

$$
\frac{1}{1+\left(v_{c}\left(T_{e}, t\right) / v\right)^{2}}
$$

where $v_{c}\left(T_{e}, t\right)$ is the time-dependent rate of inelastic collisions of the electrons of kinetic energy $T_{e}$. This collision rate amounts to several $\mathrm{THz}$ within the first nanosecond for highly energetic electrons and then decreases to the level of a few tens of MHz. Consequently, for frequencies $v$ in the $\mathrm{GHz}$ frequencies, the suppression factor can be important only during the first nanosecond, as long as the collision rate is much larger than the frequency considered for the radiation field.

\section{Expected MBR intensity at ground}

A vertical proton shower of $10^{17.5} \mathrm{eV}$ is used as a proxy to illustrate the estimation of the spectral intensity expected from MBR, to be compared to reference [1]. The parameterisation of the atmosphere selected here is the widely used US standard atmosphere, based on experimental 
data [13]. The parameterisation of the wavelength dependence of the refractive index is taken from [14]. The left panel of figure 2 shows the expected intensity at ground for different distances from the shower core. This figure is relevant for detectors such as EASIER [15], CROME [16], that sample the received radiation at ground by using regularly spaced antennas operating in the $\mathrm{GHz}$ frequency band. The right panel of figure 2 illustrates the expected intensity from an EAS with its core located at $10 \mathrm{~km}$ from the detector. This case is relevant for detectors such as MIDAS [17], or AMBER [1]. Using the same proxy shower, the presented calculation predicts a spectral intensity about 70 times lower than expected from calculations extrapolated from laboratory measurements.
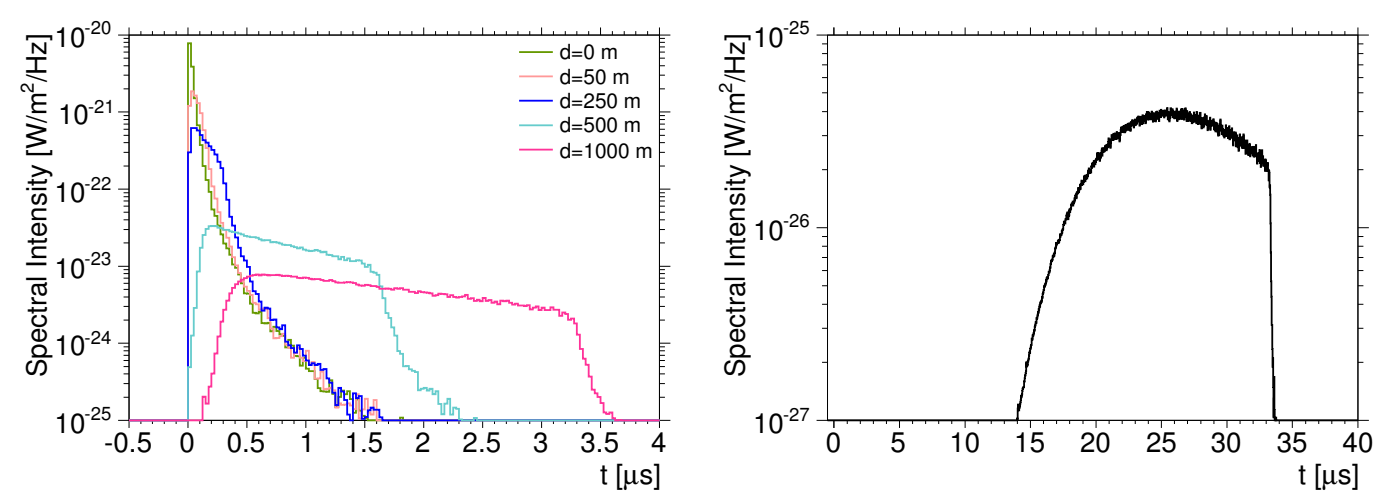

Figure 2: Left: Spectral intensity as a function of time expected at different distances from the shower core at ground level, for a vertical shower with energy $10^{17.5} \mathrm{eV}$. Right: Spectral intensity as a function of time expected at $10 \mathrm{~km}$ from the shower core at ground level, for a vertical shower with energy $10^{17.5} \mathrm{eV}$.

\section{Discussion}

Promising prospects for radio detection of air-showers using the Bremsstrahlung radiation were believed to be at hand after the announcement of measurements performed in laboratory. We have presented in this contribution a model of MBR emission from EAS predicting a much lower intensity. Lately, another laboratory measurement [18] using accelerated electrons at $95 \mathrm{keV}$ reported the detection of MBR. New installations with improved sensitivity are currently running at the Pierre Auger Observatory [15]. The improved sensitivity of this installation due to higher gains and larger effective areas of the antennas should allow the collection of a few MBR events in future years if the intensity of the emission follows our model.

There are other sources of emission but MBR susceptible to explain the few GHz events detected so far. Geomagnetic and Askaryan effects responsible for radio-emission in extensive air showers in the $\mathrm{MHz}$ frequency range have been shown to extend emission in the $\mathrm{GHz}$ range in an elliptical ring-like region around the intersection of a Cherenkov cone with its vertex at shower maximum and the ground. This is due to the coherence of the emission occurring with relative time delays with respect to the virtual front plane moving at the speed $c$ within few nanoseconds inside a thin cylinder around the shower maximum [19, 20,21]. The polarisation of the signals measured at the CROME experiment is typical of the one generated by geomagnetic emission and thus provides some support that the main process responsible for those events is the geomagnetic effect [16]. 
However, the small statistics still prevent us from drawing firm conclusions, in particular for the events detected at the Pierre Auger observatory, where both the altitude of the site and the energy threshold are higher so that the antennas are closer to the shower maximum. MBR originating from high-energy electrons in extensive air showers could also contribute significantly to the $\mathrm{GHz}$ signals during the first nanoseconds and close enough to the shower core, due to the collimation of the emitted photons along the directions of the electrons in the MeV energy range [22].

\section{References}

[1] P. W. Gorham et al., Phys. Rev. D 78 (2008) 032007

[2] I. Al Samarai et al., Astropart. Phys. 67 (2015) 26

[3] T. K. Gaisser \& A. M. Hillas, Proc. 15th ICRC (1977) Vol 8, 353

[4] K. Greisen, Prog. Cosmic Ray Physics 3 (1956) North Holland Publ.; K. Kamata \& J. Nishimura, Prog. Theor. Phys. Supp. 6 (1958) 93

[5] C. B. Opal et al., J. Chem. Phys. 55 (1971) 4100

[6] F. Arqueros, F. Blanco \& J. Rosado, New Journal of Physics 11 (2009) 065011

[7] N. Kroll \& K. N. Watson, Phys. Rev. A 5 (1972) 1883

[8] D. Rapp \& P. Englander-Golden, J. Chem. 43 (1965) 1464

[9] A. V. Phelps et al., JILA cross-sections, http://jila.colorado.edu/ avp/collision_data/electronneutral/ELECTRON.TXT

[10] V. Kas'yanov \& A. Starostin, Sov. Phys. - JETP 21 (1961) 193

[11] see e.g. L. Landau \& E. Lifchitz, Quantum Electrodynamics, Ed MIR, 1973

[12] G. Bekefi, Radiation Processes in Plasmas, John Wiley and sons, Inc., New-York, 1966

[13] NASA, NOAA and US Air Force, US standard atmosphere 1976, NASA technical report NASATM-X-74335, NOAA technical report NOAA-S/T-76-1562 (1976)

[14] R. C. Weast (ed.), Handbook of Chemistry and Physics, 67th edition, E373, The Chemical Rubber Co., Cleveland (1986)

[15] R. Gaior for the Pierre Auger Collaboration, Proc. 33rd ICRC (2013), id 0883, [arXiv:1307.5059]

[16] R. Smida et al., Phys. Rev. Lett. 113 (2014) 22, 221101

[17] J. Alvarez-Muñiz et al., Nucl. Instrum. Meth. A 719 (2013) 70

[18] E. Conti, G. Collazuol \& G. Sartori, Phys. Rev. D 90 (2014) 071102(R)

[19] J. Alvarez-Muñiz, W. R. Carvalho Jr. \& E. Zas, Astropart. Phys. 35 (2011) 325

[20] K. Werner, K. D. de Vries \& O. Scholten, Astropart. Phys. 37 (2012) 5

[21] J. Alvarez-Muñiz et al., Phys. Rev. D 86 (2012) 123007

[22] I. Al Samarai et al., in preparation 